\title{
Clamping down on exocytosis
}

DOI:

10.1038/nrn1975

\begin{abstract}
Neurotransmitter release is well known to depend on calciumtriggered excocytosis of synaptic vesicles. But what is the mechanism that couples synaptic vesicle fusion and calcium? Giraudo and colleagues report that a reversible clamping protein, known as complexin, provides the crucial link.

SNARE (SNAP receptor) proteins on the presynaptic and vesicle
\end{abstract}

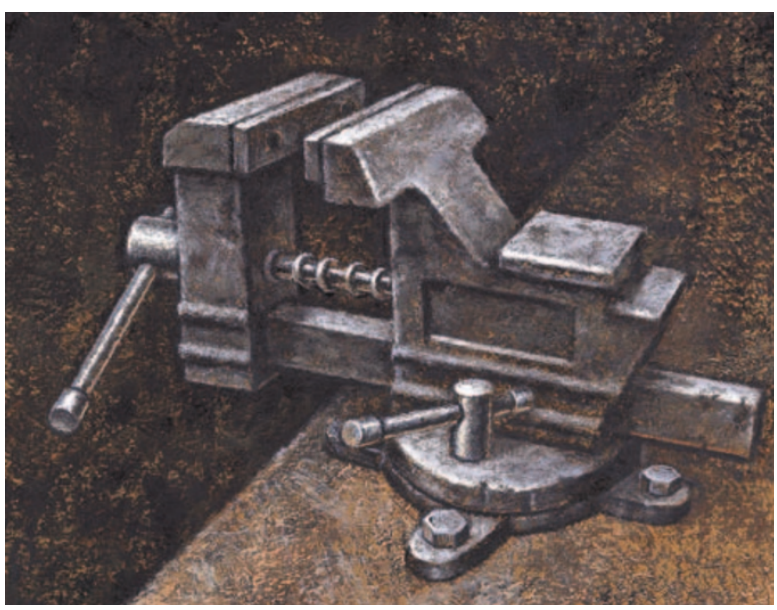

membranes form a complex that mediates vesicle fusion in response to an increase in intracellular calcium that is detected by the calciumsensing protein synaptotagmin. Complexin is known to bind to exocytotic SNARE proteins, and genetic deletion of complexin and synaptotagmin result in a similar phenotype. Complexin could therefore be a prime candidate to act cooperatively with SNARE proteins and synaptotagmin in calciummediated vesicle fusion.

Giraudo and colleagues assessed the effects of ectopically expressed SNARE proteins on the cell surface, which normally lacks the SNARE complex, to understand the role of complexin in regulating fusion. Introducing recombinant human complexin to this 'flipped' SNARE assay abolished, or 'clamped', fusion. By contrast, synaptotagmin, when added on its own or in the presence of calcium, had neither a blocking nor a stimulating effect on fusion, suggesting that it is neither a clamp nor a fusion protein. When complexin and synaptotagmin were incubated together, fusion remained blocked, but the addition of calcium in these conditions led to a full recovery.

These data are consistent with a model in which the fusion machinery is constitutively active, but is clamped by complexin during the basal state. This clamping is reversed in response to a trigger for secretion - the binding of calcium to synaptotagmin — thereby allowing fusion to take place.

Although in vivo studies will be required to confirm the clamping role of complexin in neurotransmission, this elegant work nevertheless sheds light on a key mechanism of neuronal communication that has until now remained elusive.

Alison Rowan

ORIGINAL RESEARCH PAPER Giraudo, C. G. et al. A clamping mechanism involved in SNARE-dependent exocytosis. Science 22 June 2006 (doi:10.1126/ science.1129450) FURTHER READING Rizo, J. \& Südhof, T. C. Snares and munc 18 in synaptic vesicle fusion. Nature Rev. Neurosci. 3, 641-653 (2002) 